\title{
Serial Quantitative Computed Tomography Perfusion in Aneurysmal Subarachnoid Hemorrhage
}

\author{
Cheemun Lum, Matthew J. Hogan, John Sinclair, Shane English, Howard Lesiuk, \\ Jai Shankar, Hala Ayoub
}

\begin{abstract}
Purpose: Computed tomography perfusion (CTP) has been performed to predict which patients with aneurysmal subarachnoid hemorrhage are at risk of developing delayed cerebral ischemia (DCI). Patients with severe arterial narrowing may have significant reduction in perfusion. However, many patients have less severe arterial narrowing. There is a paucity of literature evaluating perfusion changes which occur with mild to moderate narrowing. The purpose of our study was to investigate serial whole-brain CTP/ computed tomography angiography in aneurysm-related subarachnoid hemorrhage (aSAH) patients with mild to moderate angiographic narrowing. Methods: We retrospectively studied 18 aSAH patients who had baseline and follow-up whole-brain CTP/computed tomography angiography. Thirty-one regions of interest/hemisphere at six levels were grouped by vascular territory. Arterial diameters were measured at the circle of Willis. The correlation between arterial diameter and change in CTP values, change in CTP in with and without DCI, and response to intra-arterial vasodilator therapy in DCI patients was evaluated. Results: There was correlation among the overall average cerebral blood flow (CBF; $R=0.49, p<0.04)$, mean transit time $(R=-0.48, p=0.04)$, and angiographic narrowing. In individual arterial territories, there was correlation between changes in $\mathrm{CBF}$ and arterial diameter in the middle cerebral artery $(\mathrm{R}=0.53$, $p=0.03)$, posterior cerebral artery $(R=0.5, p=0.03)$, and anterior cerebral artery $(R=0.54, p=0.02)$ territories. Prolonged mean transit time was correlated with arterial diameter narrowing in the middle cerebral artery territory $(R=0.52, p=0.03)$. Patients with $D C I$ tended to have serial worsening of CBF compared with those without DCI $(\mathrm{p}=0.055)$. Conclusions: Our preliminary study demonstrates there is a correlation between mild to moderate angiographic narrowing and serial changes in perfusion in patients with aSAH. Patients developing DCI tended to have progressively worsening CBF compared with those not developing DCI.
\end{abstract}

RÉSUMÉ: Tomodensitométrie quantitative sériée avec perfusion dans l'hémorragie sous-arachnoïdienne. Objectif: Une tomodensitométrie avec perfusion (TDP) a été effectuée afin de prédire quels patients atteints d'une hémorragie sous-arachnoïdienne sont à risque de présenter une ischémie cérébrale retardée (ICR). Les patients ayant un rétrécissement artériel sévère peuvent avoir une perfusion diminuée de façon importante. Cependant, plusieurs patients ont un rétrécissement artériel moins sévère. Il existe peu de publications qui évaluent les changements de perfusion associés à un rétrécissement léger ou modéré. Le but de notre étude était d'examiner la TDP sériée du cerveau/l'angiographie par tomodensitométrie chez des patients présentant une hémorragie sous-arachnoïdienne due à un anévrisme (HSAa) et qui ont un rétrécissement de léger à modéré à l'angiographie. Méthode: Nous avons étudié rétrospectivement 18 patients atteints d'une HSAa qui ont subi une TDP du cerveau/une angiographie par tomodensitométrie. Trente et une régions d'intérêt/ hémisphère à six niveaux ont été regroupés par territoire vasculaire. Nous avons mesuré les diamètres artériels au niveau de l'hexagone de Willis. Nous avons évalué la corrélation entre le diamètre artériel et le changement des valeurs à la TDP, le changement à la TDP avec et sans ICR et la réponse au traitement par vasodilatateur artériel chez les patients présentant une ICR. Résultats: Il existait une corrélation entre le flot sanguin cérébral (FSC) moyen global ( $\mathrm{r}=0,49$; $\mathrm{p}<0,04)$, le temps de transit moyen $(\mathrm{r}=-0,48 ; \mathrm{p}=0,04)$ et le rétrécissement à l'angiographie. Dans les territoires artériels individuels, il existait une corrélation entre les changements du FSC et le diamètre artériel dans les territoires de l'artère cérébrale moyenne $(r=0,53 ; p=0,03)$, de l'artère cérébrale postérieure $(\mathrm{r}=0,5 ; \mathrm{p}=0,03)$ et de l'artère cérébrale antérieure $(\mathrm{r}=0,54 ; \mathrm{p}=0,02)$. Un temps de transit moyen prolongé était corrélé au rétrécissement du diamètre artériel dans le territoire de l'artère cérébrale moyenne $(\mathrm{r}=-0,52 ; \mathrm{p}=0,03)$. Les patients présentant une ICR avaient plus souvent une détérioration sériée du FSC par rapport à ceux qui n'avaient pas d'ICR $(\mathrm{p}=0,055)$. Conclusions: Notre étude préliminaire démontre qu'il existe une corrélation entre un rétrécissement angiographique de léger à modéré et les changements sériés de perfusion chez les patients atteints d'une HSAa. Les patients qui présentaient une ICR présentaient plus souvent une détérioration progressive du FSC par rapport à ceux qui n'en avaient pas.

Key words: Aneurysm, CT perfusion, Subarachnoid hemorrhage

doi:10.1017/cjn.2015.351

Can J Neurol Sci. 2016; 43: 375-380

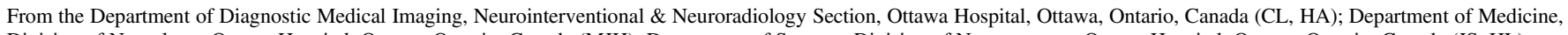
Division of Neurology, Ottawa Hospital, Ottawa, Ontario, Canada (MJH); Department of Surgery, Division of Neurosurgery, Ottawa Hospital, Ottawa, Ontario, Canada (JS, HL); Department of Medicine, Ottawa Hospital, Ottawa, Ontario, Canada (SE); Department of Radiology QEII Health Sciences Centre, Halifax, Halifax, Nova Scotia (JS). Received May 14, 2015. Final Revisions Submitted August 19, 2015.

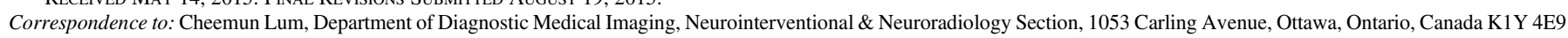
Email: chlum@ottawahospital.on.ca 
Detection of delayed cerebral ischemia (DCI) in patients with aneurysm-related subarachnoid hemorrhage (aSAH) is challenging. The clinical management of DCI varies from less invasive therapies such as hypertensive-hypervolemic treatment to more invasive intra-arterial vasodilator therapy. ${ }^{1}$ Intracranial angioplasty has been described for more severe degrees of arterial narrowing and is reported as being more durable than pharmaceutical vasodilation, but carries a higher procedural risk. $^{2}$ Knowledge of the status of cerebral perfusion at the tissue level may be helpful to determine if more aggressive therapies are warranted and to determine the efficacy of these therapies.

Computed tomography perfusion (CTP) appears to be a promising tool to detect DCI and guide therapy in patients with severe angiographic narrowing. ${ }^{3-14}$ However, available published studies using CTP suffer from numerous limitations. For example, DCI is a dynamic process, evolving over hours to days following SAH. However, most existing perfusion imaging studies provide only a single snapshot of cerebral perfusion at the time of established DCI and do not allow for serial comparisons. ${ }^{15}$ Computed tomography angiography (CTA) is now routinely performed for diagnosis and management planning of suspected aSAH. The newest generation of CT scanners allows simultaneous acquisition of CTP and CTA with a single bolus of contrast. ${ }^{16}$ The technique allows both detection of the culprit aneurysm and readily available baseline CTP, before the onset of DCI. The majority of CTP studies published were undertaken using CT scanners with limited brain coverage. The newest scanners offer whole-brain CTP. CTP performed on older scanners have different processing algorithms from newer scanners and may lead to variability in interpretation. ${ }^{17}$ Therefore, an evaluation of the relationship between arterial narrowing and changes in CTP with the current scanners capable of acquiring whole-brain CTP is necessary in patients with aSAH. There is a paucity of literature evaluating serial changes in perfusion with mild to moderate degrees of angiographic narrowing.

The primary objective of our study is to investigate the utility of whole-brain serial CTP in patients with aSAH and mild to moderate angiographic narrowing. We sought to determine if there is a correlation between mild to moderate angiographic narrowing and changes in whole-brain cerebral perfusion. Our secondary objective was to determine if patients with $D C I$ develop changes in perfusion from baseline compared to those who did not develop DCI. We also sought to investigate if changes in perfusion were associated with intra-arterial therapy for DCI.

\section{Materials ANd Methods}

The study was approved by the research ethics board of the Ottawa Hospital Research Institute and has therefore been performed in accordance with the ethical standards laid down in the 1964 Declaration of Helsinki and its later amendments. All patients gave informed consent before inclusion in this study.

\section{Patient Selection and Image Acquisition}

At our institution, patients with suspected SAH undergo a routine CTA on a 64-slice CT scanner to detect a culprit aneurysm. It was not routine to acquire baseline CTP on patients presenting initially with aSAH. Our department acquired a new

\section{Table 1: Baseline clinical and radiological data in 18 patients}

\begin{tabular}{|c|c|}
\hline & Patien \\
\hline Sex, female & 9 \\
\hline Mean age \pm SD (years) & 55 \\
\hline \multicolumn{2}{|l|}{ Admission Hunt and Hess grade } \\
\hline I & 6 \\
\hline II & 2 \\
\hline III & 3 \\
\hline IV & 4 \\
\hline $\mathrm{V}$ & 3 \\
\hline \multicolumn{2}{|l|}{ Amount of SAH (Fisher grade) } \\
\hline 1 & 1 \\
\hline 2 & 1 \\
\hline 3 & 2 \\
\hline 4 & 14 \\
\hline \multicolumn{2}{|l|}{ Treatment } \\
\hline \multicolumn{2}{|l|}{ None } \\
\hline Coil embolization & 17 \\
\hline Clip placement & 1 \\
\hline \multicolumn{2}{|l|}{ Bleeding aneurysm location } \\
\hline ICA/posterior communicating & 3 \\
\hline ACA & 9 \\
\hline MCA & 2 \\
\hline Vertebrobasilar & 4 \\
\hline
\end{tabular}

$\mathrm{MCA}=$ middle cerebral artery; $\mathrm{SD}=$ standard deviation.

320-slice CT scanner (Toshiba, Tustin, CA) capable of acquiring simultaneous whole-brain CTP and CTA data with a single contrast injection. However, this scanner is only available during daytime hours. Patients with CTA or catheter angiography evidence of a culprit aneurysm as a cause of the SAH treated by endovascular coiling or surgical clipping were retrospectively classified as aSAH. All follow-up CTP exams were following aneurysm treatment. Patients without a culprit aneurysm detected by catheter angiography were not included in the study (e.g. nonaneurysmal perimesencephalic hemorrhage). CTA and CTP was acquired with the following parameters: slice thickness $=0.5 \mathrm{~mm}$, $80 \mathrm{kV}, 100 \mathrm{~mA}$, rotation time $=1$ second, injection rate $5 \mathrm{~mL} /$ second iopamidol 370 (Bracco, Milan) with 19 image volumes obtained over 53 seconds.

We evaluated consecutive patients with aSAH who had a baseline and at least one follow-up CTA/CTP performed on our 320-slice scanner for between February 2009 and September 2010. The time interval for the study was chosen because, after September 2010, a new version of perfusion software was installed. Patients who did not have baseline CTP or without suspicion of DCI (no follow-up CTP) were excluded. Patients with significant artifacts on CTP rendering scans uninterpretable were excluded.

The following baseline characteristics were collected: sex, age, Hunt and Hess and Fisher grade, treatment, and aneurysm location (Table 1). 
A

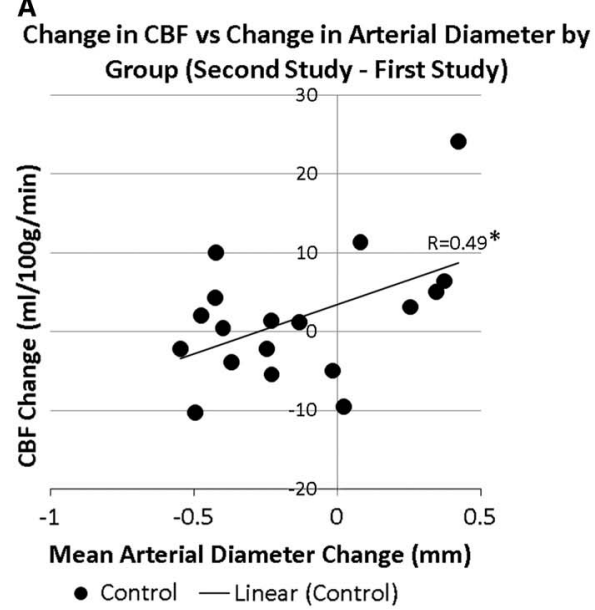

B

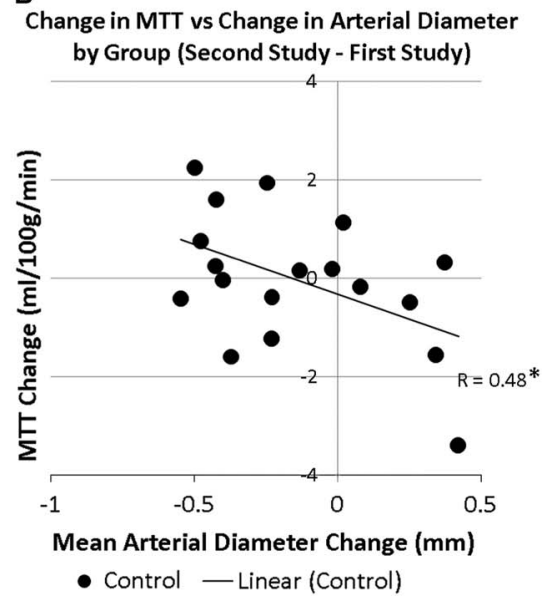

Figure 1: (A) Relationship between change in CBF and change in arterial diameter. (B) Relationship between change in MTT and change in arterial diameter.

\section{Determination of DCI}

For the purposes of this study, patients were classified retrospectively by a neurointerventionist with 12 years' experience in managing aSAH patients into those with DCI and those without DCI based on the following definition: occurrence of focal neurological impairment (such as hemiparesis, aphasia, apraxia, hemianopia, or neglect), or a decrease of at least 2 points on the Glasgow Coma Scale (either on the total score or on one of its individual components [eye, motor on either side, verbal]) lasting for at least 1 hour, the deficits were not apparent immediately after aneurysm occlusion, and could not be attributed to other causes by means of clinical assessment, CT or MRI scanning of the brain, and appropriate laboratory studies. ${ }^{18}$ Patients with suspected DCI were treated with hypervolemic hypertensive therapy and considered for intra-arterial milrinone and/or angioplasty per the institutional protocol. For severe degrees of angiographic narrowing in the supraclinoid internal carotid artery (ICA) and M1 segments angioplasty is performed at our institution. Patients with suspected DCI had CTA/CTP acquired at baseline (first study), time of suspected DCI (second study), and after intraarterial therapy (third study). Patients without DCI had baseline CTP/CTA (first study) and predischarge CTA/ CTP performed (second study).

\section{PostProcessing}

Perfusion maps were generated using a Vitrea FX 4D workstation-software version 2.0 (Toshiba). Standardized arterial and venous input regions-of-interest (ROIs) were placed over the precavernous left ICA and in the superior sagittal sinus above the torcula. The following CT perfusion maps were generated through the whole brain: cerebral blood volume, cerebral blood flow (CBF), mean transit time (MTT), and time to peak (TTP). The whole-brain CTP axial slices were reconstructed at a slab thickness of $9.6 \mathrm{~mm}$. Standardized manual ROIs were drawn over the cerebellum, posterior temporal, and occipital lobes using the midline mirror tool, covering two axial slices. For the remainder of brain, a computer-generated template of ROIs was placed in a standardized fashion in the low basal ganglia, high basal ganglia, low centrum semi-ovale, and high centrum semi-ovale levels resulting in a total of 62 ROIs over six transverse levels covering the entire brain.
Comparable axial images on serial follow-up avoiding clip or coil artifact were selected. Individual ROIs were grouped into MCA, anterior cerebral artery (ACA), and posterior cerebral artery (PCA) territories in the cerebral hemispheres and within the cerebellum based on a standardized arterial distribution map. ${ }^{19}$ Perfusion data were averaged over each group resulting in eight measured values for each perfusion parameter per study. CTP was evaluated at a separate sitting by the same observer as CTA, blinded to initial CTA results, after 6 weeks to avoid recall bias.

For this study, CTA was the modality used to assess angiographic narrowing. Arterial diameters were retrospectively evaluated from axial source and coronal MIP CTA images with an electronic caliper measurement tool applied to the CTA images using a standardized approach by a single observer with 16 years of experience in cross-sectional vascular imaging. Bilateral measurements were obtained in the supraclinoid ICA, proximal $\mathrm{M} 1$, and proximal M2, A1, A2, P1, and P2 segments. A mid-basilar measurement was also obtained. The mean arterial diameter change from baseline admission CT was calculated globally and within each arterial territory. The severity of angiographic narrowing was categorized as follows: $:^{20}$ none, $\leq 5 \%$; mild, $6 \%$ to $33 \%$; moderate, $34 \%$ to $66 \%$; and severe, $>66 \%$

\section{Statistical Analysis}

The Pearson correlation coefficient was used to determine the relationship between angiographic narrowing measured by serial CTAs and change in perfusion values. To evaluate treatment effect of intra-arterial vasodilator therapy in patients with DCI, perfusion on the posttreatment was compared with the pretreatment scan. For all patients, the change in perfusion between the first and second scan was evaluated to determine if there were significant changes in perfusion in patients who developed DCI compared with those that did not (repeated measures analysis of variance). A p value of $<0.05$ was considered significant.

\section{Results}

Over the study period, 107 patients were admitted to our institution with suspected aSAH. From these patients, 19 had both baseline and at least one follow-up CTA/CTP imaging study. 
Table 2: Pearson correlation ( $R$ value) between mean arterial diameter change and change in perfusion parameters

\begin{tabular}{|c|c|c|c|c|}
\hline & $\begin{array}{c}\mathrm{CBF} \\
(\mathrm{ml} / 100 \mathrm{~g} / \text { minute })\end{array}$ & $\begin{array}{c}\text { CBV } \\
(\mathrm{ml} / 100 \mathrm{~g})\end{array}$ & $\begin{array}{c}\text { MTT } \\
\text { (seconds) }\end{array}$ & $\begin{array}{c}\text { TTP } \\
\text { (seconds) }\end{array}$ \\
\hline \multicolumn{5}{|c|}{ By territory in all subjects } \\
\hline All territories & $0.49 *$ & 0.12 & $-0.48^{*}$ & -0.38 \\
\hline MCA & $0.53 *$ & 0.05 & $-0.52 *$ & -0.4 \\
\hline ACA & $0.54 *$ & 0.08 & -0.45 & -0.39 \\
\hline PCA & $0.50 *$ & 0.17 & -0.39 & -0.37 \\
\hline Cerebellum & 0.38 & 0.13 & -0.48 & -0.34 \\
\hline \multicolumn{5}{|c|}{ DCI vs controls } \\
\hline DCI & 0.19 & 0.15 & -0.22 & -0.62 \\
\hline Control & $0.62 *$ & 0.08 & $-0.57 *$ & -0.29 \\
\hline
\end{tabular}

$* \mathrm{p}<0.05$.

$\mathrm{MCA}=$ middle cerebral artery.

The mean decrease in arterial diameter from baseline in all DCI patients with mild angiographic narrowing was $19.6 \%$ (39 vessels). The mean decrease in arterial diameter change in all DCI patients with moderate angiographic narrowing was $48.4 \%$ (17 vessels). There were no patients in our series with severe $(>66 \%)$ angiographic narrowing. One subject was excluded because of uninterpretable CTP from a motion artifact, leaving 7 DCI and 11 non-DCI subjects $(n=18)$ for analysis (Table 1$)$. Follow-up studies were performed between days 1 and 12 after presentation. The median time (days) to the second study was 4 days (1 to 12) for DCI subjects and 4 days (2 to 12) for non-DCI subjects. One patient had the second study on day 1 as he had mild angiographic vasospasm at presentation and was suspected of having early DCI. All patients with DCI were managed with intra-arterial milrinone for symptoms of mild-to-moderate degrees of angiographic narrowing. None of the patients in this series was treated with intracranial angioplasty. One patient with DCI died and therefore did not have intra-arterial milrinone or posttreatment CTA/CTP imaging.

\section{Evaluation of the Relationship Between Perfusion Values and the Overall Mean Arterial Change}

There was a significant correlation between the overall average CBF $(\mathrm{R}=0.49, \mathrm{p}<0.04)$, MTT $(\mathrm{R}=-0.48, \mathrm{p}=0.04)$, and angiographic narrowing (Figure 1). When individual arterial territories were analyzed, there was significant correlation between changes in $\mathrm{CBF}$ and arterial diameter in the MCA $(\mathrm{R}=0.53$, $\mathrm{p}=0.03)$, PCA $(\mathrm{R}=0.5, \mathrm{p}=0.03)$, and ACA $(\mathrm{R}=0.54, \mathrm{p}=0.02)$ territories. Similarly, prolonged MTT was correlated with arterial diameter narrowing in the MCA territory $(\mathrm{R}=-0.52, \mathrm{p}=0.03)$ (Table 2). For DCI patients, there was a nonsignificant correlation of TTP with change in arterial diameter $(R=0.62, p=0.14)$. The mean arterial diameter $\%$ change in large vessels (basilar artery, $\mathrm{ICA}, \mathrm{M} 1$, or $\mathrm{A} 1)$ was $-6 \%(\mathrm{SD} \pm 38 \%)$ in the DCI group versus $0 \%$ (SD $\pm 39 \%$ ) change in the non-DCI group.

\section{Serial CTP in Patients With DCI}

Patients with DCI demonstrated a trend to decreased CBF $(p=0.055)$ when comparing the follow-up study to baseline study. In comparison, patients without DCI demonstrated an increase in $\mathrm{CBF}$. No significant difference between groups was identified for cerebral blood volume, MTT, or TTP measurements.

\section{DISCUSSION}

There is a paucity of studies evaluating mild to moderate arterial narrowing and CT perfusion changes in patients with aSAH. Our study found a correlation between mild to moderate arterial narrowing and changes in CT perfusion. The deterioration in DCI patients may be clinically more detectable in the MCA territory (focal weakness, speech and language disturbance). In our study, $\mathrm{CBF}$ and MTT were correlated with changes in arterial diameter within the MCA territory. The clinical changes, especially in patients with altered sensorium, may be more difficult to clinically evaluate. Thus, CTA narrowing and corresponding changes in perfusion in the clinical territories such as the PCA and ACA, as found in our study, may be helpful.

In our study, we found trends towards reduced CBF and prolonged TTP. One explanation for our weaker correlations with MTT and TTP may be our patients only had mild to moderate degrees of angiographic narrowing compared with other studies where more severe degrees of narrowing were more strongly correlated with decreased $\mathrm{CBF}$ and alterations in MTT and TTP. ${ }^{3-12}$ Several authors have reported threshold levels on CTP performed at the time of suspected narrowing without comparison to baseline CTP. ${ }^{3,12}$ Dankbaar found that patients with DCI had lower CBF and prolonged MTT compared with those that did not. They did not compare baseline and follow-up CT values and only evaluated perfusion at one slice. ${ }^{8}$ Sanelli found that CBF and MTT were impaired in patients with narrowing, however the mean values of CBF were generally higher (30 to $40 \mathrm{ml} / 100 \mathrm{~g} /$ minute) than our study $(20 \mathrm{ml} / 100 \mathrm{~g} /$ minute $) .{ }^{11}$ This illustrates the heterogeneity in CTP values from scanner to scanner, between vendors and difficulty with extrapolating thresholds between vendors. ${ }^{16}$ Using positron emission tomography imaging, Dar et al showed that angiographic narrowing was associated with reductions in cerebral perfusion. However, they also found that regional hypoperfusion and oligemia frequently occurred in territories and 
patients without narrowing. ${ }^{20}$ Other studies have found that flow was lower in patients and within territories affected by moderate to severe narrowing (whether proximal or distal), but there was a poor relationship between arterial narrowing and matched regional hypoperfusion. $^{21}$

Currently, to prospectively determine if a patient is at risk of developing DCI, the degree of angiographic narrowing is evaluated with transcranial Doppler, cross-sectional imaging, or catheter angiography. Previous studies using CTP have suggested that angiographic narrowing is associated with impaired perfusion. ${ }^{3-12}$ Because of the heterogeneity in CTP values from scanner to scanner, it is difficult to apply absolute threshold from others vendors. Newer whole-brain CT scanners have the attractive feature of added coverage in addition to acquisition of CTA and CTP data simultaneously with a single contrast bolus. When using older generation scanners with limited brain coverage, several slabs at the level of the basal ganglia traditionally have been processed. The benefit of whole-brain CTP is that perfusion information is available for arterial territories that may be missed if only the basal ganglia level is studied.

There are several reasons why baseline CTP may be helpful: we can compare subsequent CTP values to determine if there is a worsening in cerebral perfusion. If we see progression of angiographic narrowing in a comatose patient, it is often challenging to determine the level of risk one should undertake when considering treatment. Additional information suggesting worsening cerebral perfusion may be helpful in risk stratification and potentially guiding treatment. At times, narrowing is bilateral and severe. Therefore, comparing perfusion values to the unaffected hemisphere may not be possible. ${ }^{3}$ Very few studies have compared the evolution of changes in perfusion for patients with angiographic narrowing. Lee et al, on older generation scanners, found that group means for $\mathrm{CBF}$ at a single brain slice were significantly different in patients with no/little versus moderate/severe narrowing. ${ }^{22}$ Dankbaar compared baseline CTA but not baseline CTP to follow-up CTA and CTP in patients with DCI. ${ }^{7}$ They evaluated quantitative CTP on a single slice at the basal ganglia level and found a relationship between arterial narrowing and worsening CTP in patients with DCI. Sanelli performed CTP at baseline and when patients developed symptoms of DCI. However, their analysis only evaluated perfusion at the baseline CTP and details about evolution of CTP changes were not described. ${ }^{12}$ In our study, we were able to evaluate changes in CTP from baseline as the CTP was obtained as part of the original CTA volumetric scan. Because we were able to confirm that changes in perfusion do occur with progressive arterial narrowing, the potential to guide therapy in the future exists.

A clinical challenge exists in how best to treat patients with angiographic narrowing and DCI. In our centre, patients are followed with transcranial Doppler; however, transcranial Doppler is not always available and is user-dependent. Many of our patients have confirmatory CTAs done when DCI is suspected. When they have definite clinical symptoms, such as new weakness or aphasia, the choice to treat is clear. However, patients who are confused or comatose are difficult to examine and provide no end point for clinical examination. For this reason, serial CTP values may be useful to evaluate the temporal evolution of an individual patient's perfusion. Adding to the complexity of managing these patients, is the difference in procedural risks associated with administering $\mathrm{HH}$ therapy, intra-arterial pharmaceutical vasodilator therapy through a diagnostic catheter placed in the cervical ICA, placed with a selective microcatheter above the ophthalmic artery in the intracranial circulation or balloon angioplasty of a narrowed segment. At times, patients have severe angiographic narrowing but no clinical symptoms and may be best treated conservatively.

\section{Response to Therapy}

Nogueira reported a significant change in CBF for patients with DCI treated with IA-nicardipine. ${ }^{14}$ The CBF values performed on a different scanner were higher than our study. Haangi et al generally had lower CBF values than in our series, with no details regarding their scanner and software described. ${ }^{13}$ In our study, the perfusion tended to worsen in patients with angiographic narrowing and improve after IA therapy; however, this was not statistically significant. This may be due to the relatively small numbers in our pilot study, differences in technology, or the degree of angiographic narrowing treated. We generally reserve IA milrinone for more distal and lesser degrees of angiographic narrowing. More proximal M1 and ICA symptomatic angiographic narrowing is often treated by angioplasty at our centre. We suspect that patients with more severe angiographic narrowing would demonstrate greater changes in perfusion and greater response to angioplasty therapy than those in our current series with mild to moderate degrees of narrowing treated with vasodilators. This further emphasizes the difficulty in applying quantitative CTP values from the literature to individual patients.

\section{Risk of Radiation Exposure}

Radiation exposure with serial CTP studies has been a concern recently. ${ }^{23}$ An advantage of our protocol is our ability to obtain simultaneous CTA and CTP allowing baseline CTP to be obtained on admission. In our centre, this is particularly attractive as the decision to coil or to clip a ruptured aneurysm is determined from the initial CTA used to diagnose the aneurysm without undergoing a separate diagnostic catheter angiogram. ${ }^{24}$ For a typical CTA and CTP study on a 320 scanner, the maximum effective radiation dose was $4.6 \mathrm{mSv}$ for performed with $80 \mathrm{kV}$, $80 \mathrm{~mA}$, and 1 second per rotation, and the minimum effective dose was $3.5 \mathrm{mSv}$ performed with the same parameters. ${ }^{17}$ In our hospital, the radiation dose for a routine head CT is approximately 2.5 to $3.5 \mathrm{mSv}$, depending on the specific model of CT scanner used. Thus, the estimated radiation dose is approximately $1.3 \times$ that of a routine head CT. In comparison, the background radiation for a person living for 1 year in Boston is approximately $3 \mathrm{mSv}$, roughly equivalent to one unenhanced CT head. ${ }^{25}$ There are advantages in simultaneous CTA/CTP exams with a single contrast bolus. The radiation exposure is slightly lower than for sequential examinations that, in the past, would be required to acquire both CTA and CTP information. ${ }^{26}$ The best practice to limit radiation is to perform the fewest number of perfusion scans possible.

There are several limitations to our pilot study: relatively small sample size, relative absence of severe angiographic narrowing requiring angioplasty, retrospective nature, and variable timing of followup CTP in controls. Nonconsecutive patients were studied because of the hours of availability of the CT scanner capable of generating whole-brain CTP. Our aim is to address these issues in a larger, prospective study with inclusion of such variables as 
imaging evidence of infarction, changes in blood pressure or intracranial pressure.

In conclusion, our preliminary study evaluates serial wholebrain CTP in patients with aSAH. We confirm that mild to moderate angiographic narrowing is correlated with serial changes in whole-brain perfusion. Patients developing DCI tended to have worsening CBF compared with those without DCI.

\section{ACKNOWLEDGEMENTS}

The authors thanks H. Dabirzadeh for assistance in preparing images and Fraser Hill for assistance in preparing the manuscript.

\section{Disclosures}

None of the authors has anything to disclose.

\section{Statement OF Authorship}

CL undertook project development, data collection, and manuscript writing. MJH undertook project development, data analysis, and manuscript writing. JS, SE, HL, and JS undertook project development and manuscript writing. HA undertook data collection.

\section{REFERENCES}

1. Shankar J, P Dos Santos M, Deus-Silva L, et al. Angiographic evaluation of the effect of intra-arterial milrinone therapy in patients with vasospasm from aneurysmal subarachnoid hemorrhage. Neuroradiology. 2011;53:123-8.

2. Terry A, Zipfel G, Milner E, et al. Safety and technical efficacy of over the wire balloons for the treatment of subarachnoid hemorrhage induced cerebral vasospasm. Neurosurg Focus. 2006;21:E14.

3. Dankbaar JW, de Rooij N, Rijsdijket M, et al. Diagnostic threshold values of cerebral perfusion measured with computed tomography for delayed cerebral ischemia after aneurysmal subarachnoid hemorrhage. Stroke. 2010;41:1927-32.

4. Wintermark M, Ko NU, Smith WS, Liu S, Higashida RT, et al. Vasospasm after subarachnoid hemorrhage: utility of perfusion CT and CT angiography on diagnosis and management. AJNR Am J Neuroradiol. 2006;27:26-34.

5. Sviri GE, Britz GW, Lewis DH, Newell DW, et al. Dynamic perfusion computed tomography in the diagnosis of cerebral vasospasm. Neurosurgery. 2006;59:319-25.

6. Binaghi S, Colleoni ML, Maeder P, et al. CT angiography and perfusion CT in cerebral vasospasm after subarachnoid hemorrhage. AJNR Am J Neuroradiol. 2007;28:750-8.

7. Dankbaar JW, Rijsdijk M, van dS I, et al. Relationship between vasospasm, cerebral perfusion, and delayed cerebral ischemia after aneurysmal subarachnoid hemorrhage. Neuroradiology. 2009:51:813-9.

8. Dankbaar JW, de Rooij NK, Velthuis BK, et al. Diagnosing delayed cerebral ischemia with different CT modalities in patients with subarachnoid hemorrhage with clinical deterioration. Stroke. 2009; 40:3493-8.

9. Nabavi DG, Leblanc LM, Baxter B, et al. Monitoring cerebral perfusion after subarachnoid hemorrhage using CT. Neuroradiology. 2001;43:7-16

10. Laslo AM, Eastwood JD, Chen FX, et al. Dynamic CT perfusion imaging in subarachnoid hemorrhage-related vasospasm. AJNR Am J Neuroradiol. 2006;27:624-31.

11. Sanelli P, Ugorec I, Johnson C, et al. Using quantitative CT perfusion for evaluation of delayed cerebral ischemia following aneurysmal subarachnoid hemorrhage. AJNR Am J Neuroradiol. 2011;32: 2047-53.

12. Cremers $\mathrm{CH}$, Vanderschaaf IC, Wensink I, et al. CT perfusion and delayed cerebral ischemia in aneurysmal subarachnoid hemorrhage: a systematic review and meta-analysis. J Cerebral Blood Flow Metab. 2014;34:200-7.

13. Hänggi $\mathrm{D}$, Turowski $\mathrm{B}, \mathrm{Beseoglu} \mathrm{K}$, et al. Intra-arterial nimodipine for severe cerebral vasospasm after aneurysmal subarachnoid hemorrhage: influence on clinical course and cerebral perfusion. AJNR Am J Neuroradiol. 2008;29:1053-60.

14. Nogueira RG, Lev MH, Roccatagliata L, et al. Intra-arterial nicardipine infusion improves CT perfusion-measured cerebral blood flow in patients with subarachnoid hemorrhage-induced vasospasm. AJNR Am J Neuroradiol. 2009;30:160-4.

15. Vajkoczy P, Munch E. Vasospasm diagnosis strategies. Acta Neurochir Suppl. 2008;104:211-3.

16. Kudo K, Sasaki M, Yamada K, et al. Differences in CT perfusion maps generated by different software. Radiology. 2010;254: 200-9.

17. Murayama K, Katada K, Nakane M, et al. Whole-brain perfusion CT performed with a prototype 256-detector row CT system: initial experience. Radiology. 2009;250:202-11.

18. Vergouwen MDI, Vermeulen M, van Gijn J, et al. Definition of delayed cerebral ischemia after aneurysmal subarachnoid hemorrhage as an outcome event in clinical trials and observational studies. Stroke. 2010;41:2391-5.

19. Damassio H. A computed tomographic guide to the evaluation of cerebral vascular territories. Arch Neurol. 1983;40:138-42.

20. Dhar R, Scalfani T, Blackburn S, et al. Relationship between angiographic vasospasm and regional hypoperfusion in aneurysmal subarachnoid hemorrhage. Stroke. 2012;43:1788-94.

21. Grubb RL, Raichle ME, Eichling JO, Gado MH. Effects of subarachnoid hemorrhage on cerebral blood volume, blood flow, and oxygen utilization in humans. J Neurosurg. 1977;46:446-53.

22. Nabavi DG, LeBlanc L, Baxter B, et al. Monitoring of cerebral blood flow after subarachnoid hemorrhage using computed tomography. Neuroradiology. 2001;43:7-16.

23. Mamourian A, Young $\mathrm{H}$, Stiefel M. Cumulative radiation dose in patients admitted with subarachnoid hemorrhage. AJNR Am J Neuroradiol. 2010;31:1787-90.

24. Dabirzadeh H, Lum C, Moulton R, et al. CTA for triage of aneurysms. Cdn Assoc Radiol Meeting, Montreal, QC, 2009 (abstr).

25. Konstas A, Goldmakher G, Lee T, et al. Theoretic basis and technical implementations of CT perfusion in acute ischemic stroke, part 2. AJNR Am J Neuroradiol. 2009;30:885-92.

26. Shankar JJ, Lum C, Sharma M. Whole-brain perfusion imaging with 320-MDCT scanner: reducing radiation dose by increasing sampling interval. AJR Am J Neuroradio. 2010;195:1183-6. 